\title{
Magnetic field dependence of Delta isobars properties in a Skyrme model
}

\author{
Bing-Ran He (何秉然) \\ Department of Physics, Nanjing Normal University, Nanjing 210023, People's Republic of China
}

(Received 23 November 2018; published 20 February 2019)

\begin{abstract}
The properties of $\Delta$ isobars in a uniform magnetic field are investigated. General relations between the magnetic moments of nucleons and $\Delta$ isobars in a weak magnetic field are given. In a strong magnetic field, the masses and sizes of $\Delta$ isobars depend on the magnetic field strength in different ways: the effective masses of $\Delta^{++}, \Delta^{+}$, and $\Delta^{0}$ (the sizes of $\Delta^{++}$and $\Delta^{+}$) first decrease (increase) and then increase (decrease), whereas the effective mass of $\Delta^{-}$(the sizes of $\Delta^{0}$ and $\Delta^{-}$) always increases (decrease). Our estimates show that, in the core part of the magnetar, the equation of state for $\Delta$ isobars depends on the magnetic field, which affects the upper limit on the mass of the magnetar.
\end{abstract}

DOI: 10.1103/PhysRevD.99.034019

\section{INTRODUCTION}

The $\Delta$ isobars play a very important role in nuclear physics [1]. For example, a $\Delta$ baryon medium exists in heavy-ion colliders [2] and neutron stars [3,4]. Since $\Delta$ isobars have internal structure, the properties of $\Delta$ baryons could be dramatically changed by the strong magnetic fields that widely exist in heavy-ion colliders and magnetars [5-7]. Thus, the magnetic responses of $\Delta$ baryons could be measured in a heavy-ion collider or in a magnetar.

Besides their magnetic responses, another mystery of $\Delta$ isobars is the wide range of magnetic moments that have been experimentally measured [8,9]. Although great efforts have been made to predict the magnetic moments of $\Delta$ isobar states, many challenges still remain [10-15].

Until now, baryon properties in strong magnetic fields have been hard to predict. The difficulty is that, in strong magnetic fields, the interaction between the internal structure of a baryon and the magnetic field could be nonlinear and hard to construct without proper guidance. The Lagrangian of the Skyrme model, however, is constructed using chiral perturbation theory, guided by chiral symmetry [16], which leaves no arbitrary terms in the Lagrangian in either weak or strong magnetic fields.

In this paper, $\Delta$ isobars in a uniform magnetic field are studied in the semiclassical quantization approach of the Skyrme model [17,18]. Different wave functions for $\Delta$ isobars [19] lead to different magnetic responses. In a weak magnetic field, the general relations between magnetic

\footnotetext{
*hebingran@njnu.edu.cn
}

Published by the American Physical Society under the terms of the Creative Commons Attribution 4.0 International license. Further distribution of this work must maintain attribution to the author(s) and the published article's title, journal citation, and DOI. Funded by SCOAP. moments of nucleons and $\Delta$ isobars are obtained. Using the experimental measurements of $\mu_{p}$ and $\mu_{n}$, the magnetic moments of $\Delta$ isobars are estimated and found to be consistent with experimental results. In a strong magnetic field, it is found that when the magnetic field strength increases, the effective masses of $\Delta^{++}, \Delta^{+}$, and $\Delta^{0}$ first become lighter and then heavier, while the effective mass of $\Delta^{-}$always becomes heavier. On the other hand, the sizes of $\Delta^{++}$and $\Delta^{+}$first become larger and then smaller, whereas the sizes of $\Delta^{0}$ and $\Delta^{-}$always become smaller. Finally, since both the masses and sizes of $\Delta$ isobars depend on the strength of the magnetic field, the equation of state for $\Delta$ isobars is influenced by the magnetic field, which could affect the properties of magnetars.

\section{THE MODEL}

The minimum action of the model for our present purpose contains two parts:

$$
\Gamma=\int d^{4} x \mathscr{L}+\Gamma_{\mathrm{WZW}}
$$

where $\mathscr{L}$ represents the pion dynamics which is expressed as

$$
\begin{aligned}
\mathscr{L}= & \frac{f_{\pi}^{2}}{16} \operatorname{Tr}\left(D_{\mu} U^{\dagger} D^{\mu} U\right)+\frac{1}{32 g^{2}} \operatorname{Tr}\left(\left[U^{\dagger} D_{\mu} U, U^{\dagger} D_{\nu} U\right]^{2}\right) \\
& +\frac{m_{\pi}^{2} f_{\pi}^{2}}{16} \operatorname{Tr}\left(U+U^{\dagger}-2\right) .
\end{aligned}
$$

Here, $g$ is a dimensionless coupling constant, and $f_{\pi}$ and $m_{\pi}$ are the decay constant and the mass of the pion, respectively. The covariant derivative for $U$ is defined as 


$$
D_{\mu} U=\partial_{\mu} U-i \mathcal{L}_{\mu} U+i U \mathcal{R}_{\mu},
$$

where

$$
\mathcal{L}_{\mu}=\mathcal{R}_{\mu}=e Q_{\mathrm{B}} \mathcal{V}_{B \mu}+e Q_{\mathrm{E}} H_{\mu}
$$

for the present purpose. Here, $e$ is the unit electric charge, $Q_{\mathrm{B}}=\frac{1}{3} \mathbb{1}$ is the baryon number charge matrix, $Q_{\mathrm{E}}=$ $\frac{1}{6} \mathbb{1}+\frac{1}{2} \tau_{3}$ is the electric charge matrix, $\mathbb{1}$ is the rank 2 unit matrix, and $\tau_{3}$ is the third Pauli matrix. The external gauge field $\mathcal{V}_{B \mu}$ is associated with a $U(1)_{\mathcal{V}}$ baryon number symmetry. In the symmetric gauge, the magnetic field $H_{\mu}$ is expressed as

$$
H_{\mu}=-\frac{1}{2} B y \eta_{\mu}{ }^{1}+\frac{1}{2} B x \eta_{\mu}{ }^{2},
$$

where $\eta$ is $\operatorname{diag}(+1,-1,-1,-1)$.

The last part of the action (1), i.e. $\Gamma_{\mathrm{WZW}} \equiv \int d^{4} x \mathscr{L}_{\mathrm{WZW}}$, represents effects of the chiral anomaly, provided in Refs. [20,21].

Following Refs. [22,23], in the elliptic coordinate system, $x, y$, and $z$ are expressed as

$$
\begin{gathered}
x=c_{\rho} r \sin (\theta) \cos (\varphi), \\
y=c_{\rho} r \sin (\theta) \sin (\varphi), \\
z=c_{z} r \cos (\theta),
\end{gathered}
$$

where $c_{\rho}$ and $c_{z}$ are positive dimensionless parameters, $r \equiv \sqrt{\frac{x^{2}}{c_{\rho}^{2}}+\frac{y^{2}}{c_{\rho}^{2}}+\frac{z^{2}}{c_{z}^{2}}}$, and $\theta$ and $\varphi$ are polar angles with $\theta \in$ $[0, \pi]$ and $\varphi \in[0,2 \pi]$. In the Cartesian coordinate system, $U$ is expressed as

$U=\cos (F(r)) \mathbb{1}+\frac{i \sin (F(r))}{r}\left(\frac{\tau_{1}}{c_{\rho}} x+\frac{\tau_{2}}{c_{\rho}} y+\frac{\tau_{3}}{c_{z}} z\right)$.

In a semiclassical quantization approach $[18,24,25]$, the spin and isospin are obtained by rotating the ansatz equations (6) and (7) in both space and isospin, i.e.,

$$
\hat{U}=A(U(R)) A^{\dagger},
$$

where $A$ and $R$ are rotation matrices for isospin and spatial rotations in the $x-y$ plane, respectively. Here $A$ and $R$ satisfy

$$
\begin{gathered}
A^{-1} \dot{A}=\frac{i}{2} \omega_{a} \tau_{a}, \\
\left(R^{-1} \dot{R}\right)_{i j}=-\epsilon_{i j 3} \Omega_{3},
\end{gathered}
$$

where $a=1,2,3$ and $i, j=1,2$.
By replacing $U$ with $\hat{U}$ in the action (1), a new action is obtained,

$$
\hat{\Gamma}=\int d^{4} x\left(\hat{\mathscr{L}}+\hat{\mathscr{L}}_{\mathrm{WZW}}\right)=\int d^{4} x \hat{\mathscr{L}}_{\text {total }} .
$$

The canonical conjugate momenta of the isospin and spin are obtained by taking functional derivatives of the action with respect to $\omega_{a}$ and $\Omega_{3}$,

$$
\begin{gathered}
I_{a}=\left.\frac{\partial \hat{\mathscr{L}}_{\text {total }}}{\partial \omega_{a}}\right|_{\mathcal{V}_{B \mu} \rightarrow 0}, \\
J_{3}=\left.\frac{\partial \hat{\mathscr{L}}_{\text {total }}}{\partial \Omega_{3}}\right|_{\mathcal{V}_{B \mu} \rightarrow 0},
\end{gathered}
$$

respectively.

\section{THE GENERAL RELATIONS BETWEEN NUCLEONS AND $\triangle$ ISOBARS' MAGNETIC MOMENTS IN A WEAK MAGNETIC FIELD}

We have made calculations to the following orders in the number of colors $\left(N_{C}\right): f_{\pi} \sim \mathcal{O}\left(N_{C}^{1 / 2}\right), g \sim \mathcal{O}\left(N_{C}^{-1 / 2}\right), m_{\pi} \sim$ $\mathcal{O}\left(N_{C}^{0}\right), e B \sim \mathcal{O}\left(N_{C}^{0}\right), \omega_{a} \sim \mathcal{O}\left(N_{C}^{-1}\right)$ and $\Omega_{3} \sim \mathcal{O}\left(N_{C}^{-1}\right)$. Thus, up to $\mathcal{O}\left(N_{C}^{-1}\right)$, the Hamiltonian is obtained as

$$
\mathcal{H}=\sum_{a=1,2,3}\left(\omega_{a} I_{a}\right)+\Omega_{3} J_{3}-\left.\hat{\mathscr{L}}_{\text {total }}\right|_{\mathcal{V}_{B \mu} \rightarrow 0} .
$$

The baryon mass is obtained from

$$
M_{\Psi}=\left\langle\Psi\left|\int d V \mathcal{H}\right| \Psi\right\rangle .
$$

Here, $|\Psi\rangle$ expresses the wave functions for proton, neutron, $\Delta^{++}, \Delta^{+}, \Delta^{0}$ and $\Delta^{-}$as stated in Refs. $[18,19]$. To be specific, the masses of nucleons and $\Delta$ isobars in the weak magnetic field are given in Appendix A as examples.

The baryon magnetic moment is obtained from

$$
\mu_{\Psi}=-\frac{\partial M_{\Psi_{J_{3} \equiv J}}}{\partial(e B)} .
$$

In an extremely weak magnetic field, magnetic moments of $\Delta$ isobars are written in terms of the magnetic moments of nucleons,

$$
\begin{aligned}
\mu_{\Delta^{++}} & =\frac{3}{5}\left(4 \mu_{p}+\mu_{n}\right)+3 \mu_{I}, \\
\mu_{\Delta^{+}} & =\frac{3}{5}\left(3 \mu_{p}+2 \mu_{n}\right)+\mu_{I}, \\
\mu_{\Delta^{0}} & =\frac{3}{5}\left(2 \mu_{p}+3 \mu_{n}\right)-\mu_{I},
\end{aligned}
$$




$$
\mu_{\Delta^{-}}=\frac{3}{5}\left(\mu_{p}+4 \mu_{n}\right)-3 \mu_{I}
$$

where $\mu_{I} \equiv-\frac{E_{3}}{5 E_{2}{ }^{2}}$, and $E_{2}$ and $E_{3}$ are given in Appendix A. Here, $\mu_{I}$ can be determined numerically to be about $-0.045\left[\mu_{N}\right]$, which is much smaller than the magnitude of $\mu_{p}$ and $\mu_{n}$.

At present, there are only limited and rough results available for the magnetic moments of $\Delta$ isobars. By taking the central value of experimental results [8,9], i.e. $\mu_{\Delta^{++}}=$ $5.6\left[\mu_{N}\right]$ and $\mu_{\Delta^{+}}=2.7\left[\mu_{N}\right]$, one can verify that the experimental results satisfy the relation

$$
\frac{3 \mu_{\Delta^{+}}-\mu_{\Delta^{++}}}{\mu_{p}+\mu_{n}}=\frac{3}{1.056} \simeq 3,
$$

which is consistent with Eqs. (15).

\section{NUMERICAL RESULTS}

The equation of motion for a baryon is obtained from $\langle\Psi|\hat{\Gamma}| \Psi\rangle$ at order $\mathcal{O}\left(N_{C}\right)$. The equations of motion for $\Delta$ isobars in the weak magnetic field are given in Appendix B.

We consider a standard set of parameters for the $N_{B}=1$ sector in which $m_{\pi}=138[\mathrm{MeV}], f_{\pi}=108[\mathrm{MeV}]$, and $g=4.84[18,26]$. With no loss of generality, $c_{\rho}$ is determined to be $c_{\rho}=1 / \sqrt{c_{z}}$ [23]. For a $\Delta$ isobar state of a given $|e B|$, the parameter $c_{z}$ is fixed to minimize the corresponding $\Delta$ mass. The $|e B|$ dependence of $c_{z}$ for $\Delta$ isobar states is shown in Fig. 1. We see that a stronger magnetic field corresponds to a bigger $c_{z}$. The reason for this is that a stronger magnetic field generates a larger restriction force on a charged meson $\pi^{+,-}$in the $x-y$ plane, which stretches the shapes of $\Delta$ isobars more along the $z$ axis.

The $|e B|$ dependence of the masses of $\Delta$ isobar states is shown in Fig. 2. The curves show that by increasing the strength of the magnetic field, the masses of $\Delta^{++}, \Delta^{+}$, and



FIG. 1. $|e B|$ dependence of $c_{z}$ for $\Delta^{++}, \Delta^{+}, \Delta^{0}$ and $\Delta^{-}$.



FIG. 2. $|e B|$ dependence of $M_{\Delta^{++}}, M_{\Delta^{+}}, M_{\Delta^{0}}$, and $M_{\Delta^{-}}$.

$\Delta^{0}$ first become lighter and then heavier, whereas $\Delta^{-}$ always becomes heavier. The reason is that the Hamiltonian of $\Delta$ isobar states contains linear terms of $(e B)$ and higherorder terms of $(e B)$. The sign of the linear terms of $(e B)$ for $\Delta^{++}, \Delta^{+}$, and $\Delta^{0}$ states is different from that of the $\Delta^{-}$ state; i.e., there is a minus sign before the linear terms of $(e B)$ for $\Delta^{++}, \Delta^{+}$, and $\Delta^{0}$ states, while there is a plus sign before the linear terms of $(e B)$ for the $\Delta^{-}$state [as shown in Eqs. (A3)]. This difference causes the corresponding masses of $\Delta^{++}, \Delta^{+}$, and $\Delta^{0}$ states to decrease, and the $\Delta^{-}$state to increase, when the magnetic field is weak. Meanwhile, the higher-order terms of $(e B)$ always enhance the masses of $\Delta$ isobars, which makes their masses larger when the magnetic field is stronger.

The electric charge density for a $\Delta$ isobar state is defined as [27]

$$
\rho_{E}=\frac{1}{2} \rho_{I=0}+\left\langle\Psi\left|I_{3}\right| \Psi\right\rangle \rho_{I=1},
$$

where

$$
\begin{gathered}
\rho_{I=0} \equiv\left(\left.j_{B}^{0}\right|_{e B \rightarrow 0}\right)=\left.\frac{\partial \hat{\mathscr{L}}_{\mathrm{WZW}}}{\partial\left(e \mathcal{V}_{B 0}\right)}\right|_{\mathcal{V}_{B 0} \rightarrow 0, e B \rightarrow 0}, \\
\rho_{I=1} \equiv \frac{1}{3} \sum_{a=1,2,3} \frac{\Lambda_{a}}{\left\langle\Psi\left|\int d V \Lambda_{a}\right| \Psi\right\rangle},
\end{gathered}
$$

and

$$
\Lambda_{a} \equiv \frac{\partial^{2} \hat{\mathscr{L}}}{\partial \omega_{a}^{2}} .
$$

The $\Delta$ isobar mean square (MS) electric charge radius is defined as 


$$
\left\langle R_{\Delta}^{2}\right\rangle_{E} \equiv\left\langle\Psi\left|\int d V R^{2} \rho_{E}\right| \Psi\right\rangle
$$

where $R \equiv \sqrt{x^{2}+y^{2}+z^{2}}$.

The $|e B|$ dependence of the MS electric charge radii of $\Delta$ isobars is shown in Fig. 3. We see that for all ranges of the magnetic field strength, $\left\langle R_{\Delta}^{2}\right\rangle_{E}$ of $\Delta^{++}$and $\Delta^{+}$are always above zero, whereas for $\Delta^{0}$ and $\Delta^{-}$it is always below zero. Since the electric charges of different $\Delta$ isobars are different, the MS electric charge radii should be different. If $\Delta$ isobars do not have internal structure, the MS electric charge radii of $\Delta$ isobars should satisfy

$$
\Delta^{++}: \Delta^{+}: \Delta^{0}: \Delta^{-}=2: 1: 0:-1 .
$$

However, the electric charge of a $\Delta$ baryon is constructed by two parts: one part is related to the baryon number current density $\rho_{I=0}$ and another part is related to the isovector current density $\rho_{I=1}$. For all ranges of the magnetic field strength, the distribution of $\rho_{I=1}$ is further apart from the central point of the soliton than that of $\rho_{I=0}$. This means that MS electric charge radii of $\Delta^{++}$and $\Delta^{+}$are always above zero, whereas $\Delta^{0}$ and $\Delta^{-}$are always below zero. For example, when the magnetic field is extremely weak, i.e. $|e B| \sim 0$,

$$
\begin{gathered}
\left\langle\Delta\left|\int d V R^{2} \rho_{I=0}\right| \Delta\right\rangle=0.826\left[\mathrm{fm}^{2}\right], \\
\left\langle\Delta\left|\int d V R^{2} \rho_{I=1}\right| \Delta\right\rangle=1.8\left[\mathrm{fm}^{2}\right] ;
\end{gathered}
$$

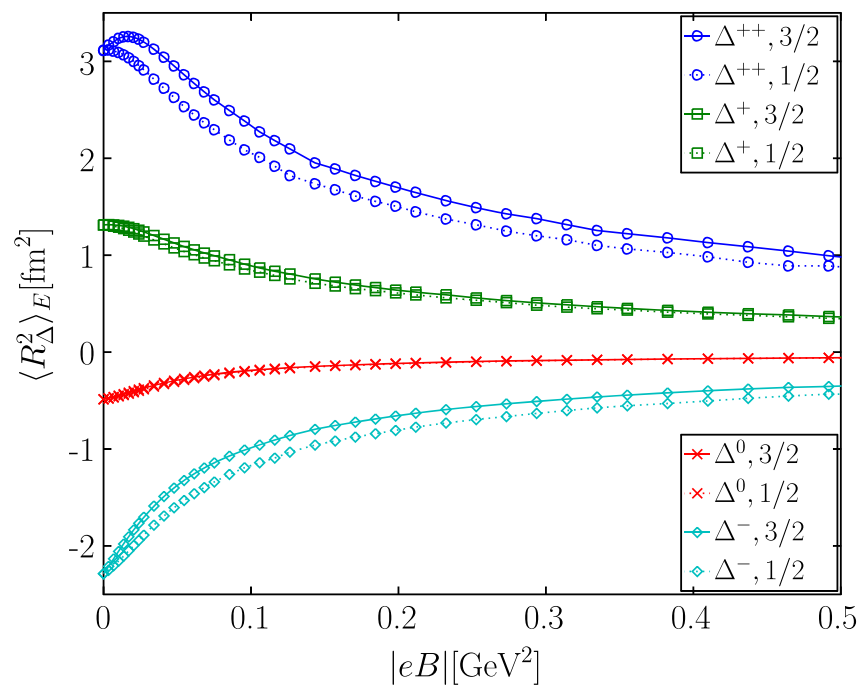

FIG. 3. $|e B|$ dependence of the $\Delta$ isobar MS electric charge radius $\left\langle R_{\Delta}^{2}\right\rangle_{E}$. thus, the MS electric charge radii of $\Delta^{++}, \Delta^{+}, \Delta^{0}$, and $\Delta^{-}$are $3.113\left[\mathrm{fm}^{2}\right], \quad 1.313\left[\mathrm{fm}^{2}\right],-0.487\left[\mathrm{fm}^{2}\right]$, and $-2.287\left[\mathrm{fm}^{2}\right]$, respectively. When the magnetic field is extremely strong,

$$
\left\langle\Delta\left|\int d V R^{2} \rho_{I=0}\right| \Delta\right\rangle \lesssim\left\langle\Delta\left|\int d V R^{2} \rho_{I=1}\right| \Delta\right\rangle .
$$

Therefore, the MS electric charge radii of $\Delta$ isobars satisfies

$$
\Delta^{++}: \Delta^{+}: \Delta^{0}: \Delta^{-} \simeq 2: 1: 0:-1 .
$$

Figure 3 also shows that, in a weak magnetic field, the magnitudes of the MS electric charge radii of $\Delta^{++}$and $\Delta^{+}$ states become larger, while that of the $\Delta^{0}$ and $\Delta^{-}$states become smaller. This fact is understandable from the equations of motion for $\Delta$ isobars shown in Eqs. (B1): there is a plus sign before the linear terms of $(e B)$ for $\Delta^{++}$ and $\Delta^{+}$states, while there is a minus sign before the linear terms of $(e B)$ for $\Delta^{0}$ and $\Delta^{-}$states. This difference leads to the result that the corresponding profile functions of $\Delta$ isobars are modified by the magnetic field, and therefore their electric charge distributions are modified. As a result, the sizes of $\Delta^{++}$and $\Delta^{+}$states increase, whereas $\Delta^{0}$ and $\Delta^{-}$states decrease when the magnetic field is weak. When the magnetic field is strong, the restriction force on the charged meson $\pi^{+,-}$in the $x-y$ plane is enhanced, and therefore the sizes of $\Delta$ isobars will be reduced.

\section{CONCLUSIONS AND DISCUSSIONS}

In this paper, the masses, magnetic moments, and MS electric charge radii of $\Delta$ isobar states in a uniform magnetic field were studied in the semiclassical quantization approach of the Skyrme model.

In the vacuum, i.e. $|e B| \sim 0$, it was shown that the magnetic moments of $\Delta$ isobars can be rewritten in terms of the magnetic moments of the proton and neutron. The comparison of our calculations of the magnetic moments for nucleons and $\Delta$ isobars with other models and experiment is shown in Table I. It shows that the theoretical predictions of $\mu_{\Delta^{++}}$and $\mu_{\Delta^{+}}$in the present model are consistent with the experimental measurements and that the relation shown in Eq. (16) can also fit for other models and experiments.

In a nonzero magnetic field, when the strength of the magnetic field increases, it was found that

(i) The masses of $\Delta^{++}, \Delta^{+}$, and $\Delta^{0}$ states first become lighter and then heavier, whereas the mass of the $\Delta^{-}$ state always becomes heavier. The minimal mass of $\Delta_{J_{3}=3 / 2}^{++}$is about $1147[\mathrm{MeV}]$ when $|e B| \sim 3.2 m_{\pi}^{2}$.

(ii) The magnitudes of MS electric charge radii corresponding to $\Delta^{++}$and $\Delta^{+}$states first become larger and then smaller, whereas the magnitudes of MS 
TABLE I. Comparison of our calculations (Skyr A) and (Skyr B) of the magnetic moments for nucleons and $\Delta$ isobars with other models and experiment (Exp.) when $|e B| \rightarrow 0$. The present work has two ways of obtaining the magnetic moments for $\Delta$ isobars: first, the experimental values of the proton and neutron magnetic moments may be taken as inputs in Eqs. (15) (Skyr A); alternatively, the masses of the nucleon and $\Delta$ may be taken as inputs to fix the model parameters (Skyr B). The other models are the Skyrme model constructed by the second and sixth order of the chiral Lagrangian [Skyr $(2+6)]$; the simple nonrelativistic quark model (NQM); and the lattice QCD (Latt.). All numbers are given in units of the nuclear magneton.

\begin{tabular}{|c|c|c|c|c|c|c|}
\hline Magnetic moment & Skyr A & Skyr B [27] & $\operatorname{Skyr}(2+6)[10]$ & NQM [11] & Latt. $[13,28]$ & Exp. $[8,9]$ \\
\hline$\mu_{p}$ & Input & 1.94 & 2.29 & Input & $3.12 \pm 0.07$ & 2.793 \\
\hline$\mu_{n}$ & Input & -1.21 & -1.70 & Input & $-1.98 \pm 0.02$ & -1.913 \\
\hline$\mu_{\Delta^{++}}$ & 5.42 & 3.80 & 4.53 & 5.56 & $3.70 \pm 0.12$ & $5.6 \pm 1.9$ \\
\hline$\mu_{\Delta^{+}}$ & 2.69 & 2.00 & 2.09 & 2.73 & $2.40 \pm 0.06$ & $2.7 \pm 3.6$ \\
\hline$\mu_{\Delta^{0}}$ & -0.05 & 0.20 & -0.36 & -0.09 & $0.001 \pm 0.016$ & \\
\hline$\mu_{\Delta^{-}}$ & -2.78 & -1.61 & -2.80 & -2.92 & $-1.85 \pm 0.06$ & \\
\hline$\frac{3 \mu_{\Delta^{+}}-\mu_{\Delta^{++}}}{\mu_{p}+\mu_{n}}$ & 3.01 & 3.01 & 2.95 & 2.99 & 3.07 & 2.84 \\
\hline
\end{tabular}

TABLE II. The density of $\Delta$ isobars when $|e B| \sim 10^{-2}\left[\mathrm{GeV}^{2}\right]$ compared to that in vacuum.

\begin{tabular}{lrr}
\hline \hline & $J_{3}=3 / 2$ & $J_{3}=1 / 2$ \\
\hline$\rho_{\Delta^{++}}$ & $-8.5 \%$ & $-0.1 \%$ \\
$\rho_{\Delta^{+}}$ & $-0.9 \%$ & $+2.4 \%$ \\
$\rho_{\Delta^{0}}$ & $-7.4 \%$ & $-5.6 \%$ \\
$\rho_{\Delta^{-}}$ & $+19.7 \%$ & $+9.8 \%$ \\
\hline \hline
\end{tabular}

electric charge radii corresponding to $\Delta^{0}$ and $\Delta^{-}$ states always become smaller.

Since both the masses and sizes of the $\Delta$ baryons depend on the strength of the magnetic field, the density of $\Delta$ baryons in the core part of the magnetar $\left(|e B| \sim 10^{-2}\left[\mathrm{GeV}^{2}\right]\right)$ can be estimated directly, as shown in Table II. Table II shows that the density of $\Delta_{J_{3}=3 / 2}^{++}$decreases by about $8.5 \%$ and the density of $\Delta_{J_{3}=3 / 2}^{-}$increases by about $19.7 \%$ compared to that in a vacuum. Thus, the equation of the state of a $\Delta$ baryon medium depends on the strength of the magnetic field, which also affects limits on the mass and size of the magnetar.

\section{ACKNOWLEDGMENTS}

This work was supported in part by the National Natural Science Foundation of China (Grant No. 11705094), Natural Science Foundation of Jiangsu Province, China (Grant No. BK20171027), Natural Science Foundation of the Higher Education Institutions of Jiangsu Province, China (Grant No. 17KJB140011), and by the Research Start-up Funding (B. R. He) of Nanjing Normal University.

\section{APPENDIX A: THE MASSES OF NUCLEONS AND $\Delta$ ISOBARS UP TO $\mathcal{O}(e B)$ IN THE WEAK MAGNETIC FIELD}

In the weak magnetic field, i.e. $|e B| \sim 0$, the shape deformation of a baryon caused by the magnetic field is negligible (as shown in Fig. 1), and thus $c_{\rho}=c_{z} \equiv 1$.
In such a case, the masses of nucleons and $\Delta$ isobars up to $\mathcal{O}(e B)$ are obtained as

$$
\begin{aligned}
& M_{p}=E_{1}+\frac{3}{8 E_{2}}+e B \frac{E_{4}}{2 E_{2}}-e B \frac{\left(E_{2}^{3}-E_{3}\right)}{6 E_{2}^{2}}, \\
& M_{n}=E_{1}+\frac{3}{8 E_{2}}+e B \frac{E_{4}}{2 E_{2}}+e B \frac{\left(E_{2}^{3}-E_{3}\right)}{6 E_{2}^{2}},
\end{aligned}
$$

$M_{\Delta_{J_{3}=3 / 2}^{++}}=E_{1}+\frac{15}{8 E_{2}}+e B \frac{3 E_{4}}{2 E_{2}}-e B \frac{3\left(E_{2}^{3}-3 E_{3}\right)}{10 E_{2}^{2}}$,

$$
\begin{aligned}
& M_{\Delta_{J_{3}=3 / 2}^{+}}=E_{1}+\frac{15}{8 E_{2}}+e B \frac{3 E_{4}}{2 E_{2}}-e B \frac{\left(E_{2}^{3}-3 E_{3}\right)}{10 E_{2}^{2}}, \\
& M_{\Delta_{J_{3}=3 / 2}^{0}}=E_{1}+\frac{15}{8 E_{2}}+e B \frac{3 E_{4}}{2 E_{2}}+e B \frac{\left(E_{2}^{3}-3 E_{3}\right)}{10 E_{2}^{2}},
\end{aligned}
$$

$M_{\Delta_{J_{3}=3 / 2}^{-}}=E_{1}+\frac{15}{8 E_{2}}+e B \frac{3 E_{4}}{2 E_{2}}+e B \frac{3\left(E_{2}{ }^{3}-3 E_{3}\right)}{10 E_{2}^{2}}$,

$$
\begin{aligned}
& M_{\Delta_{J_{3}=1 / 2}^{++}}=E_{1}+\frac{15}{8 E_{2}}+e B \frac{E_{4}}{2 E_{2}}-e B \frac{\left(E_{2}^{3}-7 E_{3}\right)}{10 E_{2}^{2}}, \\
& M_{\Delta_{J_{3}=1 / 2}^{+}}=E_{1}+\frac{15}{8 E_{2}}+e B \frac{E_{4}}{2 E_{2}}-e B \frac{\left(E_{2}^{3}-7 E_{3}\right)}{30 E_{2}^{2}}, \\
& M_{\Delta_{J_{3}=1 / 2}^{0}}=E_{1}+\frac{15}{8 E_{2}}+e B \frac{E_{4}}{2 E_{2}}+e B \frac{\left(E_{2}^{3}-7 E_{3}\right)}{30 E_{2}^{2}}, \\
& M_{\Delta_{J_{3}=1 / 2}^{-}}=E_{1}+\frac{15}{8 E_{2}}+e B \frac{E_{4}}{2 E_{2}}+e B \frac{\left(E_{2}{ }^{3}-7 E_{3}\right)}{10 E_{2}^{2}},
\end{aligned}
$$

with 


$$
\begin{aligned}
E_{1}= & \int \frac{\pi F^{\prime 2}\left(-4 \cos (2 F)+f_{\pi}^{2} r^{2} g^{2}+4\right)}{2 g^{2}} \\
& +\frac{2 \pi \sin ^{2}\left(\frac{F}{2}\right)\left(\cos (F)\left(2 f_{\pi}^{2} r^{2} g^{2}+1\right)-2 \cos (2 F)-\cos (3 F)+2 f_{\pi}^{2} r^{2} g^{2}\left(2+m_{\pi}^{2} r^{2}\right)+2\right)}{2 g^{2} r^{2}} d r,
\end{aligned}
$$

$$
E_{2}=\int \frac{2 \pi \sin ^{2}(F)\left(4 r^{2} F^{2}-2 \cos (2 F)+f_{\pi}^{2} r^{2} g^{2}+2\right)}{3 g^{2}} d r
$$

$$
\begin{aligned}
& E_{3}=\int \frac{2 \pi r^{2} \sin ^{4}(F)}{3 g^{2}} d r, \\
& E_{4}=\int \frac{r^{2} F^{\prime} \sin ^{2}(F)}{3 \pi} d r .
\end{aligned}
$$

By using the parameter set $m_{\pi}=138[\mathrm{MeV}], f_{\pi}=$ $108[\mathrm{MeV}]$, and $g=4.84$, the masses of nucleons and $\Delta$ isobars corresponding to Eqs. (A1) can be numerically determined as

$$
\begin{gathered}
M_{p}=0.939-1.033 \mathrm{eB}, \\
M_{n}=0.939+0.644 \mathrm{eB}, \\
M_{\Delta_{J_{3}=3 / 2}^{++}}=1.230-2.034 \mathrm{eB}, \\
M_{\Delta_{J_{3}=3 / 2}^{+}}=1.230-1.065 \mathrm{eB}, \\
M_{\Delta_{J_{3}=3 / 2}^{0}}=1.230-0.106 \mathrm{eB}, \\
M_{\Delta_{J_{3}=3 / 2}^{-}}=1.230+0.857 \mathrm{eB}, \\
M_{\Delta_{J_{3}=1 / 2}^{++}}=1.230-0.628 \mathrm{eB}, \\
M_{\Delta_{J_{3}=1 / 2}^{+}}=1.230-0.341 \mathrm{eB}, \\
M_{\Delta_{J_{3}=1 / 2}^{0}}=1.230-0.053 \mathrm{eB}, \\
M_{\Delta_{J_{3}=1 / 2}^{-}}=1.230+0.240 \mathrm{eB},
\end{gathered}
$$

where the masses and $e B$ are in units of $[\mathrm{GeV}]$ and $\left[\mathrm{GeV}^{2}\right]$, respectively.

\section{APPENDIX B: THE EQUATIONS FOR $\Delta$ ISOBARS UP TO $\mathcal{O}(e B)$ IN THE WEAK MAGNETIC FIELD}

In the weak magnetic field, i.e. $|e B| \sim 0$, the shape deformation of a baryon caused by the magnetic field is negligible (as shown in Fig. 1), and thus $c_{\rho}=c_{z} \equiv 1$. In such a case, the equations of motion for $\Delta$ isobars up to $\mathcal{O}(e B)$ are obtained as

$$
\begin{aligned}
& F_{\Delta_{J_{3}=3 / 2}^{++}}^{\prime \prime}=E_{5}+e B \frac{9 E_{6}}{5}, \\
& F_{\Delta_{J_{3}=3 / 2}^{+}}^{\prime \prime}=E_{5}+e B \frac{3 E_{6}}{5}, \\
& F_{\Delta_{J_{3}=3 / 2}^{\prime \prime}}^{\prime \prime}=E_{5}-e B \frac{3 E_{6}}{5}, \\
& F_{\Delta_{J_{3}=3 / 2}^{-}}^{\prime \prime}=E_{5}-e B \frac{9 E_{6}}{5}, \\
& F_{\Delta_{J_{3}=1 / 2}^{\prime \prime}}^{\prime \prime}=E_{5}+e B \frac{3 E_{6}}{5}, \\
& F_{\Delta_{J_{3}=1 / 2}^{+}}^{\prime \prime}=E_{5}+e B \frac{E_{6}}{5}, \\
& F_{\Delta_{J_{3}=1 / 2}^{\prime \prime}}^{\prime \prime}=E_{5}-e B \frac{E_{6}}{5}, \\
& F_{\Delta_{J_{3}=1 / 2}^{-}}^{\prime \prime}=E_{5}-e B \frac{3 E_{6}}{5} .
\end{aligned}
$$

Here,

$$
\begin{gathered}
E_{5}=\frac{-4 r^{3} F^{\prime}-2 r^{2} F^{2} \sin (2 F)+\sin (F)\left(\left(4 r^{2}+1\right) \cos (F)-\cos (3 F)+2 m_{\pi}^{2} r^{4}\right)}{2 r^{2}\left(-\cos (2 F)+r^{2}+1\right)}, \\
E_{6}=\frac{\sin (F)}{36\left(-\cos (2 F)+r^{2}+1\right)^{2}} \times\left(8 r F^{\prime}\left(r^{3} F^{\prime} \cos (F)+4 \sin ^{3}(F)\right)\right. \\
\left.-4 m_{\pi}^{2} r^{4} \cos (2 F)+\left(4 r^{2}+3\right) \cos (3 F)-2\left(4 r^{4}+2 r^{2}+1\right) \cos (F)-\cos (5 F)+4 m_{\pi}^{2} r^{4}\right) .
\end{gathered}
$$


[1] G. Cattapan and L. S. Ferreira, Phys. Rep. 362, 303 (2002).

[2] S. Typel and H. H. Wolter, Nucl. Phys. A656, 331 (1999).

[3] E. E. Kolomeitsev, K. A. Maslov, and D. N. Voskresensky, Nucl. Phys. A961, 106 (2017).

[4] J. J. Li, A. Sedrakian, and F. Weber, Phys. Lett. B 783, 234 (2018).

[5] D. E. Kharzeev, K. Landsteiner, A. Schmitt, and H. U. Yee, Lect. Notes Phys. 871, 1 (2013).

[6] V. A. Miransky and I. A. Shovkovy, Phys. Rep. 576, 1 (2015).

[7] V. Skokov, A. Y. Illarionov, and V. Toneev, Int. J. Mod. Phys. A 24, 5925 (2009).

[8] C. Patrignani et al. (Particle Data Group), Chin. Phys. C 40, 100001 (2016).

[9] M. Tanabashi et al. (Particle Data Group), Phys. Rev. D 98, 030001 (2018).

[10] J. H. Kim, C. H. Lee, and H. K. Lee, Nucl. Phys. A501, 835 (1989).

[11] E. E. Jenkins and A. V. Manohar, Phys. Lett. B 335, 452 (1994).

[12] V. Pascalutsa, M. Vanderhaeghen, and S. N. Yang, Phys. Rep. 437, 125 (2007).
[13] C. Aubin, K. Orginos, V. Pascalutsa, and M. Vanderhaeghen, Phys. Rev. D 79, 051502 (2009).

[14] M. D. Slaughter, Phys. Rev. D 84, 071303 (2011).

[15] A. Kaur and A. Upadhyay, Eur. Phys. J. A 52, 105 (2016).

[16] I. Zahed and G. E. Brown, Phys. Rep. 142, 1 (1986).

[17] T. H. R. Skyrme, Nucl. Phys. 31, 556 (1962).

[18] G. S. Adkins, C. R. Nappi, and E. Witten, Nucl. Phys. B228, 552 (1983).

[19] S. M. H. Wong, arXiv:hep-ph/0207194.

[20] J. Wess and B. Zumino, Phys. Lett. 37B, 95 (1971).

[21] E. Witten, Nucl. Phys. B223, 422 (1983).

[22] G. Holzwarth and B. Schwesinger, Rep. Prog. Phys. 49, 825 (1986).

[23] B. R. He, Phys. Rev. D 92, 111503(R) (2015).

[24] E. Braaten and L. Carson, Phys. Rev. D 38, 3525 (1988).

[25] S. Krusch, Ann. Phys. (Amsterdam) 304, 103 (2003).

[26] G. S. Adkins and C. R. Nappi, Nucl. Phys. B233, 109 (1984).

[27] B. R. He, Phys. Lett. B 765, 109 (2017).

[28] S. R. Beane, E. Chang, S. Cohen, W. Detmold, H. W. Lin, K. Orginos, A. Parreño, M. J. Savage, and B. C. Tiburzi, Phys. Rev. Lett. 113, 252001 (2014). 